\title{
Strategy Research on Intensive Design of Cultural Building Complex
}

(Architecture Design-China Radio \& TV International Architecture Design-Research Institute, 100045, China)

\begin{abstract}
1 Introduction
From the process of urban development in the modern world, the development of cultural arts has become an important trend for the world's cultural development, and the integration of cultural arts into the urban building design is also an unavoidable trend of future urban building development. Single building is already unable to meet the requirements of people. The people no longer just pursue a building that serves as a sheltered spot; they even more pursue a comfortable, pleasant, green and healthy living environment. The construction of spiritual civilization of a city encourages more and more architects to consider how to better fuse the buildings they design into the city for better embodiment of the culture of the city.
\end{abstract}

\section{Concepts of Intensive Design of Cultural Building Complex}

\subsection{Characteristics}

In a certain city space, different buildings are combined on a planning basis to form a cultural building complex. Generally, the cultural building complex utilizes the public space to integrate different buildings in a way that the connections between each building are effectively enhanced, thus forming a building group also called a building complex. The cultural building complex is usually located in the center part of the city and a bond connecting each and every building in the city, highlighting the cultural and spiritual civilization of a city and having iconic influences on the development of the city. The intensive design of cultural building complexes can effectively improve the quality of overall environment in buildings and allow for a further increase in the integral image of the city.

Different from general building complexes, the cultural building complex has some unique characteristics. Firstly, the size of the cultural building complex is normally far bigger than the buildings of other forms. It typically combines multiple cultural buildings, at the same time, the combination way will be more compliant with the features and laws of the development of a city; secondly, the cultural building complex is an interconnected and united integral and it echoes the development environment in the entire city; thirdly, the cultural building complex is built through high-level design of inner space.

Meanwhile, the cultural building complex has some characteristics in culture. First, it will combine multiple cultural functions which are different from each other and supplementary with each other. Second, it is a symbol and a delivery of spiritual civilization of a city. Third, it always keeps in mind the importance of culture and takes culture as the background and theme in building design, highlighting the embodiment force of the culture. Fourth, it does not have such high density as general building groups. It only has the characteristic of intensification.

\subsection{Functions and Spatial Features}

\begin{abstract}
With the development of social economy and the constant advancement of technology, there are big changes in buildings and great progress in architectural thought and ideals in the cities around our country. Intensive development of cultural building complex is a building method that has emerged in the field of architecture over the past few years. This method can make urban buildings to be not only safe and effective, but also more consistent with the philosophy and environment care requirements of the development of the whole city and allow residents to live in a comfortable and healthy city, by using more sensibly arranged urban space, through scientific rationalized planning and combination with characteristics of local urban development. This paper mainly starts with the intensive design of cultural building complexes and discusses how to adopt strategies to drive better development of cultural building complexes.
\end{abstract}

Key words: Cultural building complex; Design strategy; Intensification

Published online: $30^{\text {th }}$ Nov, 2017 
The functions and spatial features of the cultural building complexes are as follows: First, the cultural building complex is reasonably built and fully equipped. It basically can meet the requirements of all people and is able to better play its role without causing waste or shortage of resources. For example, Fed square in Melbourne is such successful case. Second, the architectural characteristics of cultural building complexes are integrated, without the phenomenon of several irrelevant buildings showing up in the group, taking the cultural building as an aim of design. Third, the cultural building complex also has personalized unique characteristics. Although with the same theme, each building expresses unique architectural cultures and styles, such as Riverside Park of Yangzi River. Fourth, spatial intensification is one of its most distinguishing cultural characteristics. It maximizes the planning of space in a limited space and makes the best of each inch of space. Meanwhile, some cultural building complexes are also filled with personalized characteristics, adhere to the thought of human orientation, fully consider the feelings of people, and build themselves into a unique cultural building complex through combination with characteristics like transportation status, geographical location and climate environment.

\section{Design Principles of Cultural Building Complex}

The design principles of cultural building complex is to combine multiple buildings with different attributes and functions in a public space to form a large-scale interconnected building group characterized by intensification, which is generally built in the center position of the city and has influences and driving roles in each and every aspects of the periphery. Intensive design has many advantages. It contributes to the effective use and development of urban resources when large-scale construction is undergone and can reasonably utilize limited space to construct buildings, without generating excessive spaces and allowing the arrange of many irregular buildings to occupy many spaces that cannot be effectively used, beneficial to the optimization of architectural environment in the city. It not only can promote the development of economy and the prosperity of society, but also can push the evolution of modernized cities, increase the utilization rate of spaces, reduce the waste of rare resources like land and enable the arrangement and planning of cities to be optimized. At the same time, multi-functional architectural design provides users with convenient and quick services. One-stop services can create overall results and cluster effect of "One plus on more than two", which is also similar with many phenomena in actual life, for example, in China, many merchandisers who sell products of the same type generally will gather in the same region, and this is the benefit of cluster effect.

Since World War II, especially China's reform and opening-up, urban architectures have developed rapidly with the extensive presence of high buildings and mansions, and the development trend gradually shifts to intensive development from dense development, with the increasing pursuit of multiple functions and comprehensive development of buildings instead of simple display of individual building functions. Many buildings simultaneously have a number of functions like shopping, catering, recreation, culture, experience, life and even work and can effectively utilize the public space to realize their role and maximization of services.

\section{Strategies of Intensive Design of Cultural Building Complex}

\subsection{Diversified Design of Cultural Buildings}

The cultural building complex is typically built in the central position of the city, normally including multiple cultural elements. The mutual fusion and mutual supplementation of multiple cultures results in a cultural building complex. Generally, this cultural building will not singly choose only one culture, which otherwise is already out of line with the requirements of cultural development for architectures; and normally it will chose mutual fusion of multiple cultures with urban development characteristics. But since multiple cultures are combined, the phenomenon of difficult integration and cultural overlap will likely occur, intensive design is needed to compound multiple cultures effectively. Generally, the architectural type of cultural building complexes can be divided into the following four categories:

\subsubsection{Cultural Building Complex Focused on Public Exhibition}

This type of complex mainly is to show people all the historical cultural elements of cities by exhibiting the development overview of such cities. The specific implementation method is to build historical museums and exhibition halls etc., which effectively combine all the cultural characteristics of local cities and place them in the same space to be displayed. Meanwhile, there are also buildings, such as cultural theatre and opera house, in cities, which that form another demonstration form of cultures. The two forms are dependent on and supplementary with each other, allowing for further development of the culture building complex.

\subsubsection{Cultural Building Complex Focused on Performance}

In addition to using cultural building complexes focused on public exhibition, adopting cultural building complexes focused on performance forms like expression language, action and music is also possible. This way is very attractive and apt to draw interest and attention from people, delivering cultures to people through such performance form.

\subsubsection{Cultural Building Focused on} Cultural Display and Cultural Performance

This type of building is to combine two kinds of cultural buildings, not only including cultural display, but also including cultural performance forms. In such buildings, spaces like studio, library and culture showroom are generally included, with cultural performance forms being mores concrete and diversified.

Besides the above three cultural building complexes, there are multi-element compound cultural building complexes, with integrations of multiple cultures, having strong shocking effect and leaving deeper impression on people.

\subsection{Intensive Design of Building}

\subsubsection{Integrated Layout Design}


The architectural method is a method, based on which, in construction, layout and planning are made according to the integral building, and different functions and roles are designed to leave the whole structure relatively coordinated and united and becoming a comprehensive building.

\subsubsection{Series-type Layout Design}

In this type of architectural design, the layout of spatial structures is relatively scattered, each space is connected and distinguished relying on a long corridor, and the orientation of each space is also arranged and designed in accordance with the direction of the long corridor to distinguish front and rear positions and primary and secondary positions.

\subsubsection{Decentralized Layout Design}

In this type of layout design, the arrangement of each small branch is conducted mainly according to the integrity of cultural buildings to define the relationship between each building, outstandingly express the most characteristic and iconic portions and appropriately lower similar cultural display of other portions.

\subsection{Narrative Design of Building Space}

The cultural building complex not only better expressed the historical development process of cities, regional culture, and urban spiritual civilization, but also makes a scientific planning on building space in a narrative way, enables visiting objects to fuse time and space with this technique and feel the cultural connotations expressed by the building, gets visitors to conduct memory association and realizes expression of urban cultural subjects. The narrative technique normally includes the following categories:

4.3.1 The Combination of Time and Space with the Method of Expression

In this expression mode, two scattered spatial structures are put together into the same space. For this mode, nice ideology of ancient times is normally combined and reasonably fused into modern architecture, to include ancient classic beauty and modern simplification and fashion in the building, with both perfectly integrated, fully reflecting cultural connotations of builders and highlighting cultural characteristics of the city.
4.3.2 Realize Reproduction of Scenarios with Narration Interposed Techniques

This method is a method in which an independent person or a thing is interposed into a range of persons or things with the same characteristics or development laws to enable it to better express the emotion and though that the builder want to express and give people a fresh view and an innovative feeling without causing unexpected and unnatural feelings. For example, in the cultural centers in some cities, intensive design philosophies will be utilized to design regions that comply with the living philosophy of urban residents and carry out perfect fusion and exchanges of human beings and things. As another example, in the design of Yantai Cultural Center, the museum' $s$ logo of "Convergent Sea, Cultural Rocks" was specially designed, with the styling of several huge rocks, activating the whole spatial system, maximizing the available space of the city and embodying the thought of human orientation and the infinite wisdom of the designers.

\subsubsection{Conduct Memorization and Descriptions on History with Flashback Techniques}

This method is the most commonly used means in current urban cultural buildings. With the method, the history and development process of the city are highlighted and described, and the longitudinal development of the city is prolonged and expanded. For example, in Walker Art Center, this demonstration way was adopted, with the fusion of new and old elements seen everywhere. The design of the new center not only keeps the essence of the old one, but also connects streets in the city with transparent glasses, integrating the building into urban life quietly.

\subsubsection{Edit Historical Clips with Leapfrog Techniques}

This method can better drive the emotion of visiting objects, allow visitors to deeply experience and feel the culture, and reach the goal of intensifying the cultural building complex. For example, Harbin Grand Theatre was designed with the shell as a shaping prototype to reflect the Songhua River culture of Harbin. The building lines, which like moving clouds and flowing water, travel and stroll in sea like a shell, perfectly embodying the urban culture of Harbin, the northern ice city.

\section{Conclusion}

In general, the cultural building complex is the important symbol and characteristics of a city and also the carrier in the cultural inheritance of a city, which can form a closely linked, capacious and composite sharing space in the unban space to agglomerate the culture and spiritual civilization of a city and is an integral of urban culture and even an important part of world cultures and human civilization history. In today when urbanization process develops rapidly, the intensification of cultural building complexes has been an import development trend and has a better development prospect. In this building mode, it is possible to make urban buildings to be not only safe and effective, but also more consistent with the requirements of philosophies and environment care of urban development and allow people to live in a comfortable and healthy urban life by using more reasonably arranged urban spaces through scientific rationalized planning and combination with the characteristics of development of local cities. When intensive design is made, firstly, it is required to figure out the concept and characteristics of intensification of cultural building complexes and identify the design principle of cultural building complexes, which are the most basic requirement that needs to be mastered for a designer or a builder. Next, it is required to figure out how to make intensive design of cultural building complexes, which is a materialized process of action and implementation for thoughts and ideas, a process that needs to be constantly examined and accumulated in practice. Whether the theory is feasible and whether the thought is reasonable and scientific is known only through actual operation. It is possible to make the created cultural building complex to play its role in the city and to be able to form a comprehensive cultural space in the city, inherit and carry forward the city's historical culture and construction of spiritual civilization, enrich people's spiritual and cultural life and promote cultural construction of the whole city, the whole country and even the whole world only if the ideas and design principles in which the intensive design is involved are firstly figured out and understood, and then effective implementation 
strategies are adopted, and finally the intensive design of buildings is made. It is hoped that the discussions in this paper could offer some helps in the intensive design of cultural building complexes and drive better development of cities.

\section{References}

[1] Wang Jiafeng, Han Juan, Zhao Yanping. Brief Discussion on the
Design for Exterior Decoration of Modern Building [J].Shaanxi Architecture,2013 (08):36-37.

[2] Li Gang. Design Thinking for Corporate Office Space [J].Science \& Technology Economy Market, 2014 (11):49-50.

[3] Jia Dong. Inheritance of Traditional Elements in Modern Building Design [J]. China Homes (issue for the end of the month), 2013(07):148-149.

[4] Guo Xiangzhen. Analysis on Urban Building Complex [J].Morden Property
Management (Issue for the beginning of the month),2014,(07).

[5] Wei Yichun, Xiang Ke. Research on the Combination Mode of Culture and Commerce in Cultural Building Complex [J].Huazhong Architecture, 2015, (12).

[6] Wang Nasi, Zhang Ali. Analysis on the Design of Building Complex [J].China Packaging Industry,2013,(16). measures for cracks in high rise buildings $[\mathrm{N}]$. Yili daily (Han), 2007-11-13 (006) 